\title{
ON EMPLOYING A SAVITZKY-GOLAY FILTERING STAGE TO IMPROVE PERFORMANCE OF SPECTRUM SENSING IN CR APPLICATIONS CONCERNING VDSA APPROACH
}

\section{Leopoldo Angrisani'), Domenico Capriglione' ${ }^{2)}$, Gianni Cerro' ${ }^{2)}$, Luigi Ferrigno ${ }^{2)}$, Gianfranco Miele ${ }^{3)}$}

\author{
1) University of Naples Federico II, Department of Electrical Engineering and Information Technology, Claudio 21, 80125 Naples, Italy \\ (angrisan@unina.it) \\ 2) University of Cassino and Southern Lazio, Department of Electrical and Information Engineering, G. Di Biasio 43, 03043 Cassino, Italy \\ (capriglione@unicas.it, $\bowtie$ g.cerro@unicas.it,+3907762993683,ferrigno@unicas.it) \\ 3) University of Salerno, Department of Business Studies and Research (Management \& Information Technology) - SIMASLab, \\ Giovanni Paolo II 132,84084 Fisciano, Italy (gmiele@unisa.it)
}

\begin{abstract}
In this paper, a filtering stage based on employing a Savitzky-Golay (SG) filter is proposed to be used in the spectrum sensing phase of a Cognitive Radio (CR) communication paradigm for Vehicular Dynamic Spectrum Access (VDSA). It is used to smooth the acquired spectra, which constitute the input for a spectrum sensing algorithm. The sensing phase is necessary, since VDSA is based on an opportunistic approach to the spectral resource, and the opportunities are represented by the user-free spectrum zones, to be detected through the sensing phase. Each filter typology presents peculiarities in terms of its computational cost, de-noising ability and signal shape reconstruction. The SG filtering properties are compared with those of the linear Moving Average (MA) filter, widely used in the CR framework. Important improvements are proposed.
\end{abstract}

Keywords: Cognitive Radio, Smoothing filter, VDSA, Spectrum Sensing, Spectral Measurements.

(C) 2016 Polish Academy of Sciences. All rights reserved

\section{Introduction}

In recent years, the road traffic was significantly increasing, thus making the traditional transport network unsuitable for the traffic volume and average speeds of modern vehicles. Most roads were constructed a long time ago, when traffic requirements were substantially different. Lastly, the traffic increase does not correspond to the road renovation rate.

Consequently, a pressing need to deal with is keeping roads safe and increasing user routing efficiency. To this aim, many national governments started to support the Intelligent Transportation System (ITS), in order to compensate the lack of efficient roads with the ability to move along them in a smarter way. ITS is defined by the European Council in the directive 2010/40/EU [1] as "advanced applications of information and communication technologies to the road transport sector in order to improve environmental performance, energy efficiency, safety and security of road transport, including the transport of dangerous goods, public security and passenger and freight mobility". According to the definition, the crucial role is played by Vehicle-to-Vehicle (V2V) and Vehicle-to-Infrastructure (V2I) communications. Taking into account the first category (V2V), since the vehicles travel at a very high speed, the communication needs short delays to be reliable [2].

Finally, the extreme mobility does not enable well exploiting traditional communication infrastructures, where mobile terminals refer to fixed base stations, but a Peer-to-Peer (P2P) paradigm seems to be preferable. The best achievement of the international standard authorities 
toward this direction is the IEEE 802.11p standard, also known as Dedicated Short-Range Communication (DSRC), intended for Vehicular ad-hoc Networks (VANETs). Currently, this is the only standard supporting the direct Vehicle-to-Vehicle (V2V) communication. The FCC (Federal Communication Commission) has allocated 7 channels, $10 \mathrm{MHz}$ wide, in the band 5.850-5.925 GHz [3-5]. Some problems affect this standard: frequency hopping in moving vehicles [6], and Doppler Effect, provoking dangerous frequency shifts; considering two vehicles, approaching each other at $130 \mathrm{~km} / \mathrm{h}$, the frequency shift is about $1.42 \mathrm{kHz}$. If the OFDM modulation technique is employed, the communication can be definitely corrupted by such a frequency change. In order to improve the communication and avoid such phenomena, the research community is moving toward a different solution, mainly focusing on the possibility of working at lower frequencies, mainly under $1 \mathrm{GHz}$, where Doppler Shift is considerably lower and the communication range can be significantly extended.

Due to the goodness in terms of the communication range, such a frequency interval has been chosen by most technologies in the field of wireless communications, making it heavily overcrowded. Fortunately, many studies have demonstrated that several portions of the radio spectrum are not in use for significant periods of time [7,8], enabling to exploit such bands in a more dynamic way, i.e. to use the silent periods of the license owners to deploy secondary services in the same frequency interval. Among them, the primary role is surely played by the Vehicular Dynamic Spectrum Access (VDSA) approach. VDSA combines two main advantages: achieving high spectrum efficiency, thanks to the dynamic access to the spectral resource, and its suitability for high mobility patterns such as the road traffic [2]. The first goal can be achieved if there is accurate knowledge of the electromagnetic context around the VDSA device zone. Therefore, spectrum sensing techniques need to be implemented, in order to find out the frequency occupation intervals.

In the literature, there are many well-assessed techniques addressing this issue, such as energy detection [9], cyclostationarity-based detection [10], matched-filtered detection [11] and others [12]. Stemming from the past experience of the authors in PSD estimation and spectral analysis [13-15], in this paper, a well-functioning edge detection spectrum sensing method presented in [16], based on the frequency domain analysis and originally developed for CR applications, is employed as the testing algorithm of the main aim of the paper - the filtering stage.

In fact, one of the critical issues of each spectrum-sensing algorithm is represented by its precision, in terms of revealed bands. In order to address the issue, the paper proposes a signal conditioning stage in which the acquired trace is de-noised using the Savitzky-Golay (SG) filter [17-19], which has been rarely employed - instead of the widely used Moving Average (MA) filter [20] - as the de-noising stage in a cognitive system. The goal is to expose the actual improvement in the frequency detection error (later called $\varepsilon$ ), obtained after the sensing stage with respect to the standard moving average usage. Moreover, as reported in Section 3, the new filtering approach enables the user to tune up more Degrees of Freedom (DoFs) than the linear MA does; such an approach makes it suitable for different signal shapes and conditions.

As for the organization of the paper, the spectrum sensing method under test is briefly described in Section 2 and some theoretical hints on the considered de-noising techniques are provided in Section 3. In Section 4, two performance evaluation stages are presented: firstly, in the simulation environment, where scenarios are generated by Matlab ${ }^{\mathrm{TM}}$ software; secondly, in the emulation environment, where the same scenarios are executed by using laboratory instrumentations. Conclusions follow in Section 5. 


\section{The spectrum sensing method under test}

Let $B$ be the frequency interval of the analysed radio spectrum, available for supporting several wireless telecommunication systems. Each transmitter can use different modulation techniques and operating frequencies, and occupy different bandwidths inside them.

The method aims to identify the portions of the radio spectrum that are currently in use either by a Primary User (PU) or by another transmitter. In Fig. 1, a block diagram of the spectrum sensing method is presented. It is an edge detection algorithm in the frequency domain.

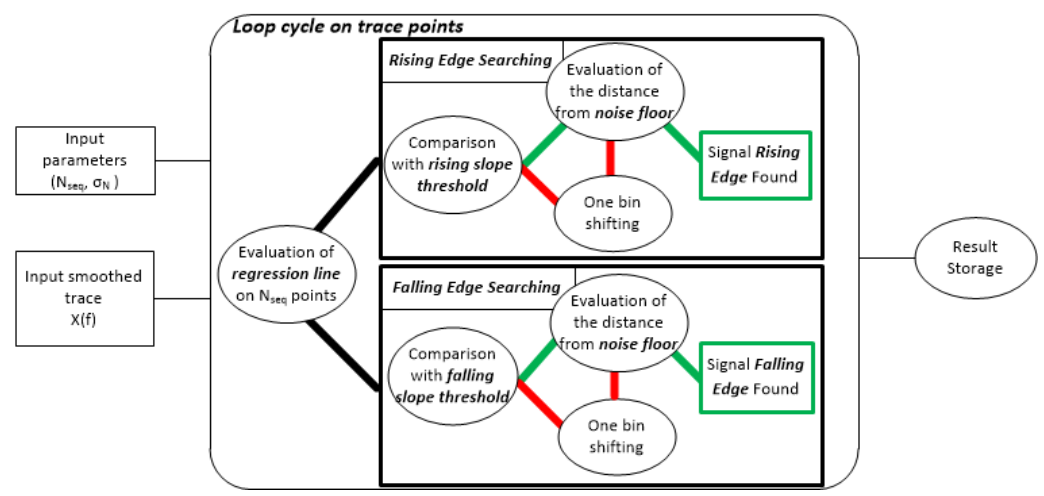

Fig. 1. A block diagram of the spectrum sensing method under test.

As the input parameters, $N_{\text {seq }}$ and $\sigma_{N}$ represent the sample window size on which edges have to be detected and the noise standard deviation, respectively. The other input is the filtered spectrum, in order to reduce noise in the trace. Such an operation can be performed using many different kinds of filters: a widely-used one is the linear Moving Average (MA) filter; despite its simplicity it shows critical problems in terms of sharpness preservation of the signals edges, as explained in the following section. To overcome the problem, the Savitzky-Golay smoothing method [11] is proposed and analysed in the presented work as a filtering stage used before the sensing phase. After de-noising, the algorithm evaluates the Noise floor $\left(N_{f l}\right)$ of the smoothed trace. Then, the occupied portions of the frequency interval are discovered by estimation of the spectrum edges.

In order to find edges, a regression line on $N_{\text {seq }}$ samples is computed. Its slope is compared with a proper value (the rising/falling slope threshold, depending on the demanded edge). At the beginning of the loop cycle, the algorithm has to find the starting rising edge: only the upper part of the diagram is executed. When the edge is found, the method switches to the lower part, looking for the falling edge. When the latter is also found, the frequency bandwidth enclosed between the rising and falling edges is declared as occupied. Please note that the edges need further verifications before being declared as the signal edges. In particular, the rising edge is detected only if the distance between the amplitude of the last bin of the analysed window and the $N_{f l}$ value is greater than $3 \sigma_{N}$, in order to get a $99.7 \%$ probability to deal with the user signal rather than noise, under the assumption of having Additive White Gaussian Noise (AWGN) in the analysed spectrum. Similarly, to be sure that the correct falling edge has been detected, another comparison between the last bin amplitude and $N_{f l}$ is performed. In this case, the last bin amplitude must be comparable to the $N_{f l}$ value. The first version of the sensing method used in this paper has been presented in [16]. 


\section{Some notes on the considered filtering techniques}

\subsection{The Savitzky-Golay filter}

The SG filter [17] is a method for data smoothing, based on polynomial approximation of the raw data in a fixed data window. SG filters have two DoFs: the polynomial order and the window length. The first parameter enables choosing the degree of the smoothing process, which is inversely proportional to the order: the higher the polynomial order, the better the smoothing data follow the raw data, with the advantage of retaining the edges and the disadvantage to follow noise fluctuations, too. The second DoF, the window length, has the advantage, if wide, of neutralizing the high frequency noise contribution, whose fluctuations are smoothed by the polynomial fitting; on the other hand, a wide window is very risky because if a narrowband signal is present, it is clearly smoothed and there is no possibility to detect it in the sensing phase. To give analytical hints to the problem, for a given $n$-degree-polynomial, the algorithm looks for the best " $n+1$ " polynomial coefficients in order to fit the raw data in the best way, and evaluates the result in the central point of the window.

In order to maintain symmetry, the window length must be odd, so that the evaluation point had the same number of points on its right and left. The window is then one-bin-right shifted and the same fitting process is repeated, by finding new coefficients and evaluating the resulting polynomial in the central bin again. In the choice of best coefficients, a Least Mean Square algorithm is applied. It is possible to prove that such an approach gives the same results as considering a tantamount impulsive response of a proper low pass filter. In such a way, the computational complexity becomes lower, because the impulsive response can be computed once, and just a convolution with the entry data needs to be evaluated. An optimization approach to choosing the best parameters for employing such a filter is reported in [19]. In the following, the authors will choose a correlation approach to establish the best parameters for using in SG smoothing operations.

\subsection{The linear Moving Average (MA) filter}

The linear MA filter [20] is widely known and used for its simple implementation and low computational cost, the desired features for low complexity devices as Cognitive Radios. The filter shows some other important peculiarities: it is able to reduce random noise and to keep a sharp step response, in some working conditions. In its linear variant, the only parameter to be set is the window length. The operating principle is trivial: performing the averaging process on samples of the selected window, one-bin-right shifting, repeating the average on new data.

Because of the described operations, it presents an important disadvantage: the resulting array is right-shifted by the half of the moving window length. There is a trade-off choice to use this filter category: the wider the length, the greater the noise reduction and the smaller the sharpness of the edges, which results in heavier frequency errors.

On the other hand, if one chooses a shorter window length, the effect of noise reduction is lighter, whilst the edge sharpness is enhanced. For VDSA's applications, it is important to keep very sharp edges to reach the highest possible precision in detecting the occupied bands in the spectrum portion of interest; at the same time, it is also mandatory to strongly reduce the noise contribution, in order to be effective also for very low SNRs (Signal to Noise Ratios).

In Fig. 2 the outputs of the described filtering methods are compared. 


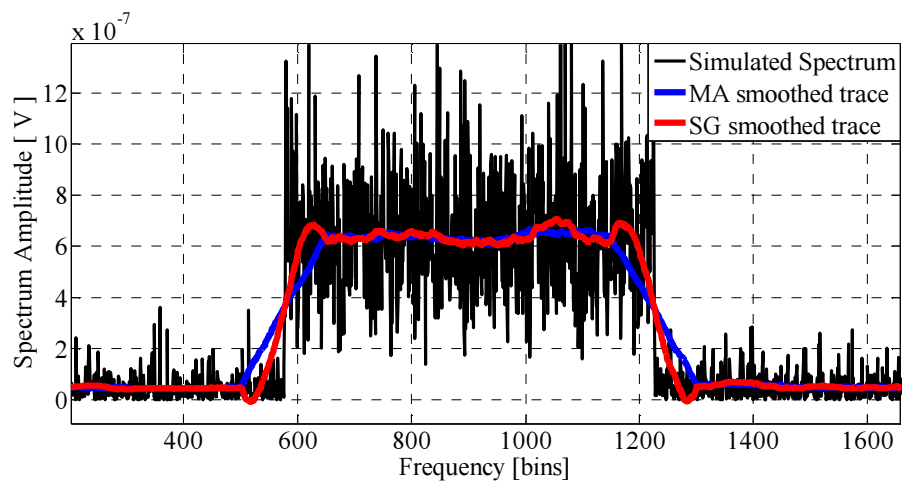

Fig. 2. Comparison of the filtering effects of the Moving Aaverage (MA) and Savitzky-Golay (SG) methods.

\section{Results}

\subsection{The simulation environment}

In this section, the results of tests carried out in a simulation environment are reported. The simulation software developed in Matlab ${ }^{\mathrm{TM}}$ environment has been employed and some test scenarios have been considered. In all of them, the performance of the edge detection spectrum sensing algorithm has been tested, using both MA and SG filters, in order to make a comparison between them.

Moreover, the results have been also validated using the emulation scenarios, which reflect the same features as the simulation ones, evaluating how the method can be effectively employed in real scenarios. The emulation results will be shown in the following section.

\subsubsection{Description of the proposed scenarios}

The choice of some particular scenarios is based on the spectrum of interest for CR applications and, especially, for VDSA communication. In particular, sub-GHz frequencies are the best core to be exploited to optimize opportunistic access to the spectral resource. In this frequency range, the most promising and usable frequency bands are VHF (174-230 MHz) and UHF (470-862 MHz) which are usually occupied by two main sources: wireless microphones and DVB-T signals. Accordingly, several scenarios have been realized by simulating the presence of such users. An observation interval of $48 \mathrm{MHz}$ has been considered. It has been subdivided into 2401 frequency bins in order to have a frequency resolution equal to $20 \mathrm{kHz}$ : the wireless microphone signal spectrum (bandwidth $=200 \mathrm{kHz}$ ) occupies 10 bins, while the DVB-T signal spectra occupy 381 bins $(8 \mathrm{MHz})$ and 335 bins $(7 \mathrm{MHz})$.

Table 1. The technical summary of the presented scenarios.

\begin{tabular}{|c|c|c|c|c|c|}
\hline Scenario ID & Users & Fc $_{1}[\mathbf{b i n s}]$ & $\mathbf{B}_{1}[\mathbf{b i n s}]$ & $\mathbf{F c}_{2}$ [bins] & $\mathbf{B}_{2}$ [bins] \\
\hline DVB-T1 & 1 & 1700 & 381 & $/$ & $/$ \\
\hline WIR-MIC & 1 & 1175 & 10 & $/$ & $/$ \\
\hline DVB-T2 & 2 & 400 & 335 & 1000 & 335 \\
\hline HYBRID & 2 & 500 & 10 & 2100 & 381 \\
\hline
\end{tabular}




\subsubsection{Figures of merit}

Evaluation of performance is based on two figures of merit:

1. Correlation coefficient $(\rho)$ : it is defined, for two given arrays $X$ and $Y$, as follows:

$$
\rho(X, Y)=\frac{\operatorname{Cov}(X, Y)}{\sqrt{\operatorname{Var}(X) \operatorname{Var}(Y)}},
$$

It is useful to test and give a numerical value of the likelihood between two different vectors: in this work, it is used for estimating the degree of filtering of the smoothing methods, as it will be described in the simulation results.

2. Frequency error $(\varepsilon)[\%]$ : it is evaluated for the starting and ending frequencies:

$$
\varepsilon_{\text {start }}=\frac{1}{N} \sum_{i=0}^{N} \frac{\left(f_{t, i}-f_{e, i}\right)}{B} 100 \%, \varepsilon_{\text {stop }}=\frac{1}{N} \sum_{i=0}^{N} \frac{\left(f_{e, i}-f_{t, i}\right)}{B} 100 \%,
$$

where $f_{t, i}$ is the imposed start/stop frequency at the $i$-th iteration, while $f_{e, i}$ is the estimated start/stop frequency obtained at the output of the sensing algorithm; $B$ is the signal bandwidth. The simultaneous presence of two indexes $\left(\varepsilon_{\text {start }}, \varepsilon_{\text {stop }}\right)$ to provide the same information is aimed for keeping the coherence of meaning for the detected bandwidth. They are both positive when the sensing algorithm detects the rising edge before the imposed one and the falling edge after its real position: the detected bandwidth is thus wider than the real one. On the other hand, they are negative when the edge is detected inside the true signal bandwidth and, consequently, the revealed bandwidth is narrower than the real one.

\subsubsection{Results in the simulation environment}

For the sake of brevity and similarity of results, only the DVB-T1 and HYBRID scenarios will be discussed.

The correlation results for the DVB-T1 scenario are shown in Figs. 3 and 4. In particular, the Pearson Correlation Coefficient $(\rho)$ has been chosen as a similarity index, to estimate how effective can be the filter application in both noise removal and shape preservation. The analysis has been carried out on 500 trials for each condition, in terms of SNRs and scenarios. More specifically, the tested SNRs are within $[-15,5] \mathrm{dB}$ range, with the aim of finding the lowest bound of signal detection, for which the filtering stage does not improve performance. The positive correlation results have been obtained for SNR values of down to $-10 \mathrm{~dB}$ (see Fig. 5) for almost all conditions, after filtering the spectra with the proposed method.

As for filter parameters, the observation window ranges from $1 \%$ to $10 \%$ of the entire tested span, while the polynomial order, concerning SG filtering, belongs to the set $[1,10]$. Every figure regarding correlation is made up of two subfigures. The upper one is the mean value of $\rho$, whereas the lower one is the standard deviation $(\sigma)$ of the obtained values over the repeated trials.

It is possible to observe that $\rho$ is generally proportional to SNR, while its behaviour regarding the observation window is not predictable and is strongly influenced by the analysed scenario. As for $\sigma$, it is inversely proportional to SNR: the lower SNR, the higher the relative weight it has on the mean value, i.e. the results become less reliable and the average value is nothing but a rough estimate of its real value. Moreover, considering the behaviour of $\sigma$ with respect to the filtering window, a decreasing tendency is observed for positive SNRs, whereas it is virtually independent for negative SNRs $(-10 \mathrm{~dB})$.

The MA filtered spectra show a lower correlation value with respect to SG, which is able to better reconstruct the signal for medium/high SNRs. 
For low SNRs (Fig. 5), the mean values and standard deviations are comparable, so considerations on trends are not reliable, and they can be better estimated by analysing the $\varepsilon$ error.

In Figs. 6 and 7, results for the HYBRID scenario are presented. Wireless microphone and DVB-T transmitter signals are simultaneously present in the tested spectrum. As previously said, the dependence between observation window and correlation, for a given SNR, is changed. Other considerations on the correlation behavior with respect to SNR are still valid, even if correlation seems to keep constant values for the homologous points at $5 \mathrm{~dB}$ and $0 \mathrm{~dB}$. Moreover, from the figures, it is possible to state that for narrower filtering windows (less than $4 \%$ ), which are used mostly when information about the average bandwidth of the users is not available, SG filters show a higher correlation value with respect to MA.
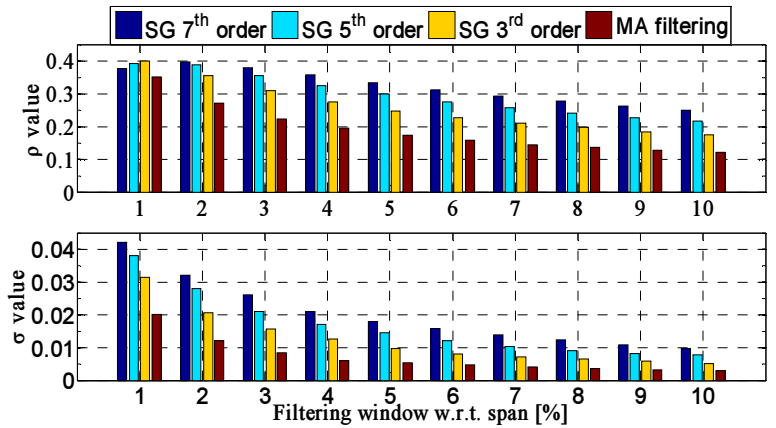

Fig. 3. Mean values and standard deviations of the $\rho$ value for DVB-T1 scenario at $5 \mathrm{~dB}$ SNR.

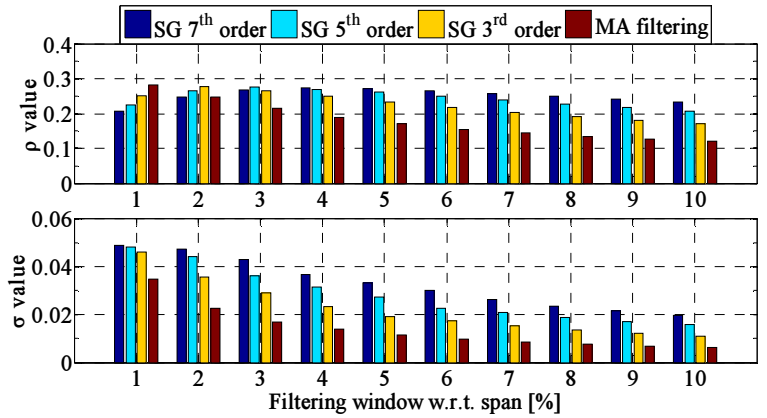

Fig. 4. Mean values and standard deviations of the $\rho$ value for DVB-T1 scenario at 0dB SNR.
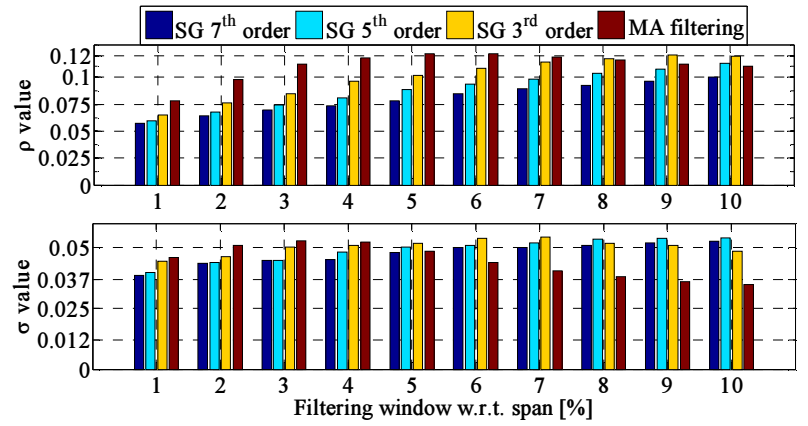

Fig. 5. Mean values and standard deviations of the $\rho$ value for DVB-T1 scenario at $-10 \mathrm{~dB}$ SNR. 

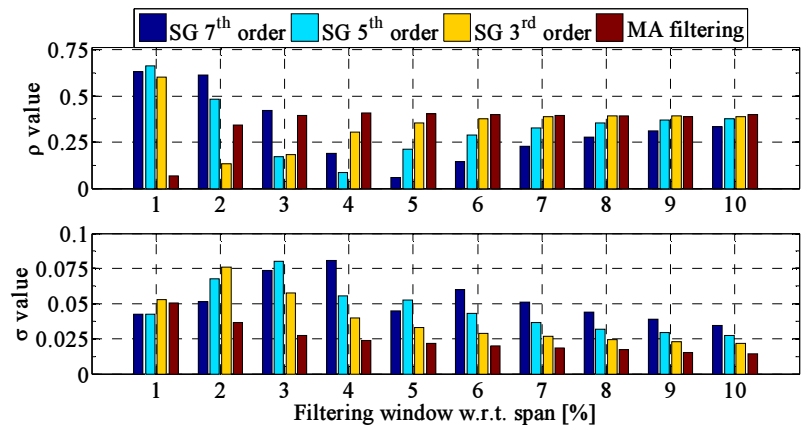

Fig. 6. Mean values and standard deviations of the $\rho$ value for HYBRID scenario at $5 \mathrm{~dB}$ SNR.

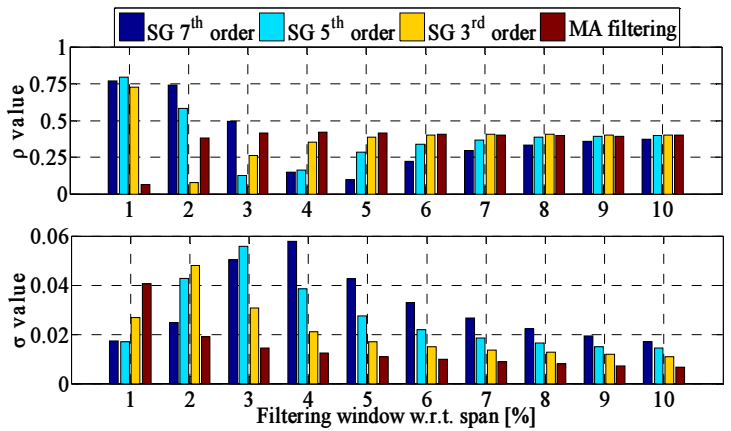

Fig. 7. Mean values and standard deviations of the $\rho$ value for HYBRID scenario at $0 \mathrm{~dB}$ SNR.

Considering the results of $\varepsilon$ errors, it is necessary to clarify the meaning of this figure of merit: a negative error value means that the detected starting/stopping frequency (the output of sensing algorithm) is inside the true occupied bandwidth. On the contrary, when the error value is positive, the algorithm is detecting a frequency bin outside the occupied bandwidth. So, a negative error is much worse than a positive one, since it means that the algorithm is detecting the user signal in a partial way and, consequently, it is stating that the remaining spectrum portion is free, while it is actually occupied.

A positive error, on the other hand, means only that the algorithm is overestimating the true bandwidth; therefore the cognitive terminal can exploit smaller spectral resource than the available one. According to the non-interference principle, negative errors have to be avoided or minimized.

A possible approach to fix the problem could be the choice to transmit in a band remaining far enough from the detected band, in order to be sure that no interference could occur.

In Fig. 8, the frequency errors for the DVB-T1 scenario are presented. It clearly shows different error levels resulted from both filtering methods. The legend indicates $\varepsilon_{\text {start }}$ as the starting frequency bin error of the occupied bandwidth, while $\varepsilon_{\text {stop }}-$ as the stopping frequency bin error of the bandwidth. For SNR values greater or equal to $-5 \mathrm{~dB}$, the SG filtered spectrum output error is almost negligible, so the detection is much more accurate than the one being the result of the MA filtering. Moreover, the MA errors are usually negative, while the SG errors are essentially positive, meaning that the band is lightly overestimated, which represents a good condition for sensing, since no interference will harm the licensed users. 


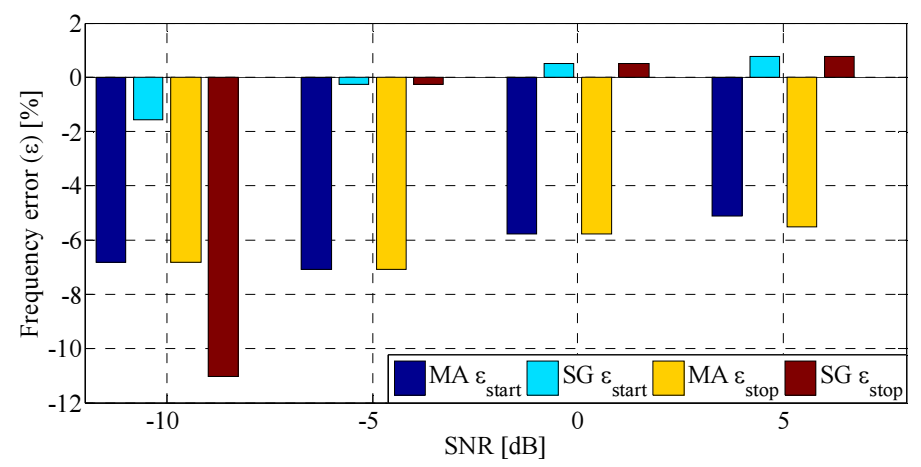

Fig. 8. The frequency error results for the DVB-T1 scenario.

a)

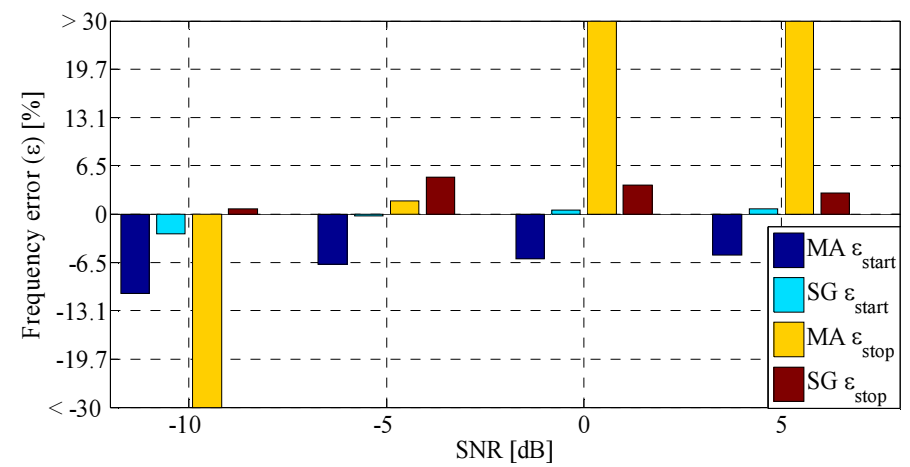

b)

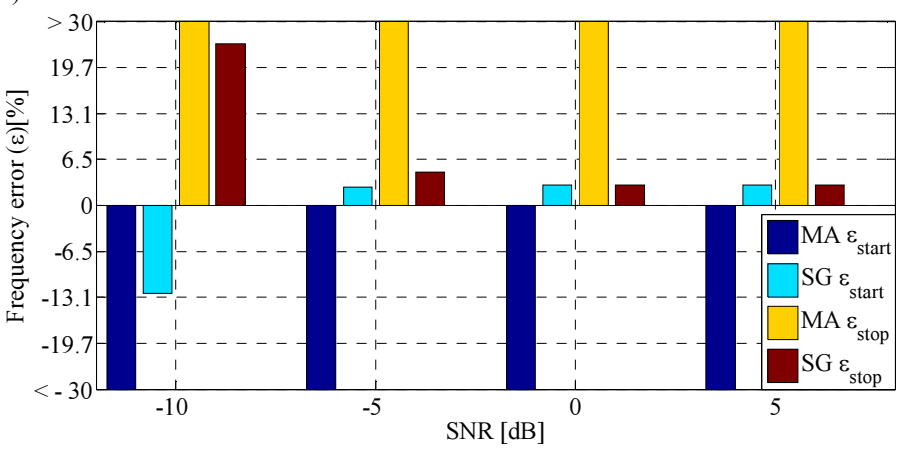

Fig. 9. The frequency error results for the HYBRID scenario: a) a DVB-T transmitter; b) a wireless microphone.

In Figs. 9a and 9b, the results for the HYBRID scenario are provided. The scenario includes wireless microphone and DVB-T transmitter signals. Firstly, it should be noted that when "> 30 " or " $<-30$ " is displayed in the figure, it is conventionally said that the algorithm has not been able to identify the signal edges accurately, i.e. the output is not coherent with the true signal bandwidth, providing frequency edges not localized in the neighbourhood of the user signal. What happens is clear: the MA approach does not enable correct detection of the wireless microphone, considering it just as noise spikes, whereas the SG approach enables it with very small and acceptable errors. 
Figure 9a presents detection of the DVB-T transmitter signal in the same scenario. In this case, things are better for both approaches, but the MA on some occasions still meets problems with detection of the falling edges, since they are almost at the end of the spectrum of interest and - consequently - some filtering problems cause incorrect detection. When SNR is equal to $-5 \mathrm{~dB}$, as an isolated case, the detection is also enabled with the MA filtering approach.

Generally, SG gives - for almost all SNRs - better frequency error values, keeping them positive and small. However, this filtering has a small drawback: the detection is precise only when the algorithm provides an output. When SNR decreases down to a very low ratio, the SG filtering is not so suitable for reducing noise, because polynomial fitting is able to follow the data trend and, consequently, if noise is predominant, noise samples are followed. In such a case, anyway, the MA filtering does not enable detection either.

For medium/high SNRs (greater or equal to $-5 \mathrm{~dB}$ ) the SG is absolutely to be preferred, as shown in the previous figures.

\subsection{The emulation environment}

\subsubsection{Description of the arranged measurement set-up}

Using an experimental setup, the scenarios presented in Table 1 have been executed.

The following instruments composed the setup:

1. Vector Signal Generator (VSG) Agilent N5182A MXG, the output frequency range: 100 $\mathrm{kHz}-6 \mathrm{GHz}$;

2. Real-Time Spectrum Analyser (RSA) Tektronix RSA6114A, the input frequency range: 9 $\mathrm{kHz}-14.0 \mathrm{GHz}$

3. PC laptop, Intel i7 processor @ 2.4 GHz, 8 GB RAM.

The VSG has been driven by the laptop using Agilent Signal Studio for Digital Video ${ }^{T M}$ ("PHASE 1"); the coaxial cable connection between VSG and RSA (in order to compute the FFT of the time domain signal) is described in "PHASE 2", while the acquisition process from RSA to PC has been executed by the NI LabVIEW ${ }^{\mathrm{TM}}$ software - it is presented in "PHASE 3".

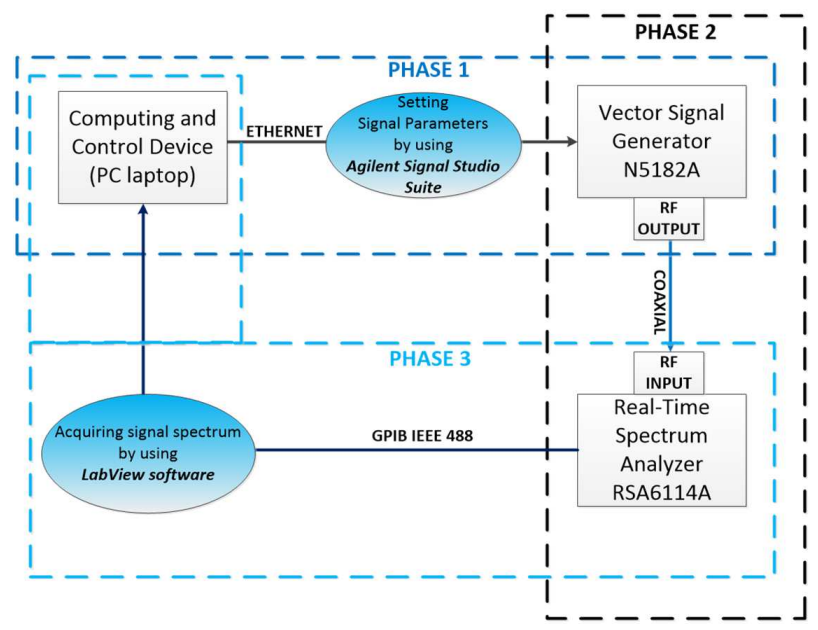

Fig. 10. A block diagram of the experimental setup. 


\subsubsection{Results in the emulation environment}

To show similarity of the correlation results in the simulation and emulation environment, only the results regarding the DVB-T1 scenario are presented in the following figures. Since considerations are similar, they are not repeated. On the contrary, the frequency errors are shown to underline some differences.

Looking at the results shown in Fig. 11, it is possible to state that they are very similar to those shown in Fig. 3, even if the correlation values are lower. This phenomenon is due to the instruments and connections, which are not ideal and, consequently, heavily affected by noise contribution. It means that it is more difficult to be able to reconstruct a signal in the emulation environment, and the experienced "SNR wall" in the simulation context has to be considered higher for the emulation and, consequently, real contexts.

Stability of repeated MA trials is maintained, as well as the tendency of increasing the correlation value by an increment of the polynomial degree of the SG filter.

The correlation results are better observed in Fig. 12. Linear MA and 5th order SG filters are applied to the noisy spectrum. Signal edges are clearly more effectively retained by the SG than by the MA.

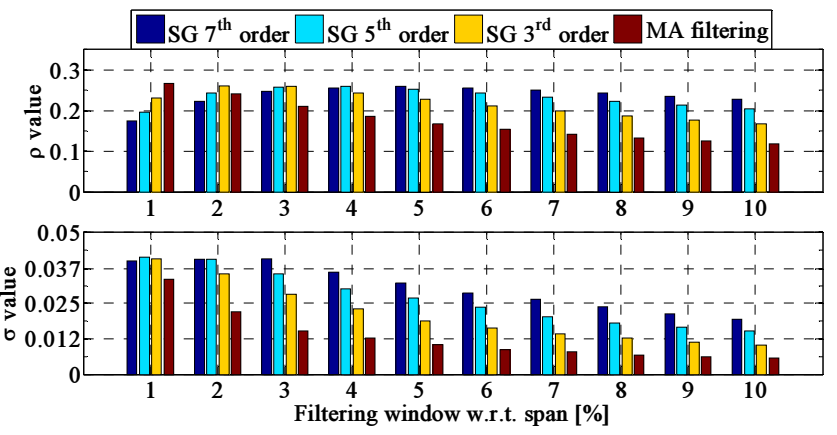

Fig. 11. Mean values and standard deviations of the $\rho$ value for DVB-T1 scenario at $5 \mathrm{~dB}$ SNR in the emulation environment.

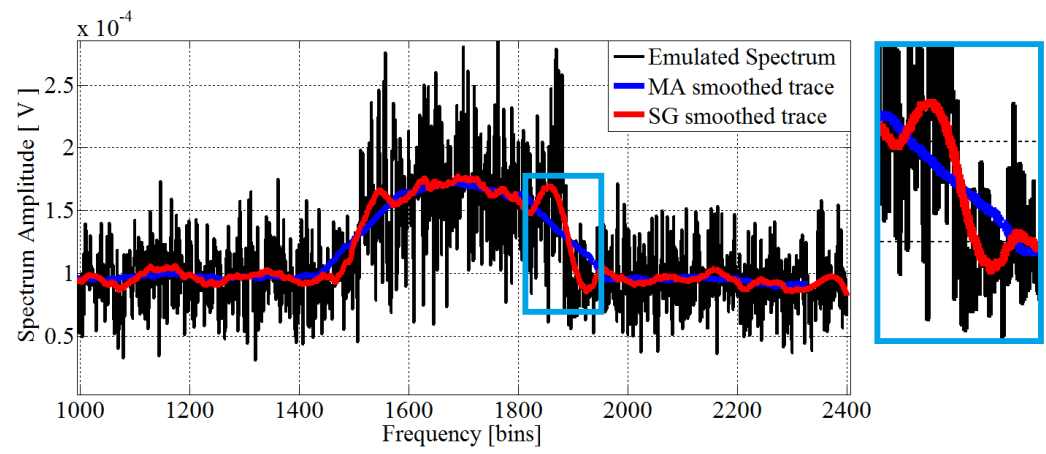

Fig. 12. The emulated filtered spectrum at $-5 \mathrm{~dB}$ SNR. 


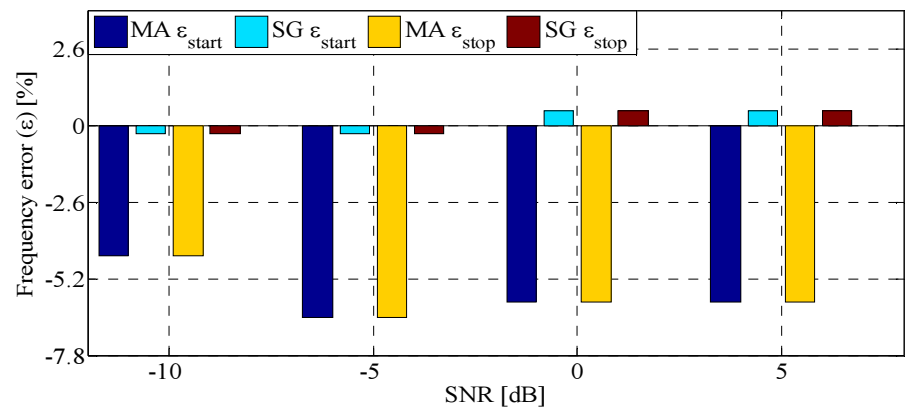

Fig. 13. The frequency error results for DVB-T1 scenario in the emulation environment.

Lower correlation values mean also worsening a performance in the frequency error, as shown in Figs. 13, 14a, 14b. While in the DVB-T1 scenario there are no substantial differences between the simulation and emulation results (it is possible to see even a light improvement in detection), the situation gets worse for the HYBRID scenario.

Due to the noise corruption, a wireless microphone is actually undetectable for SNR values less or equal to $-5 \mathrm{~dB}$, without distinction between the $\mathrm{SG}$ and MA approaches. The core is the right part of Fig. 14b, where it is explained how the SG approach becomes suitable for detecting the wireless microphone in a precise way for higher SNRs, while the MA approach does not. Therefore, also in the emulation environment, the improvement resulting from using the SG is notable.

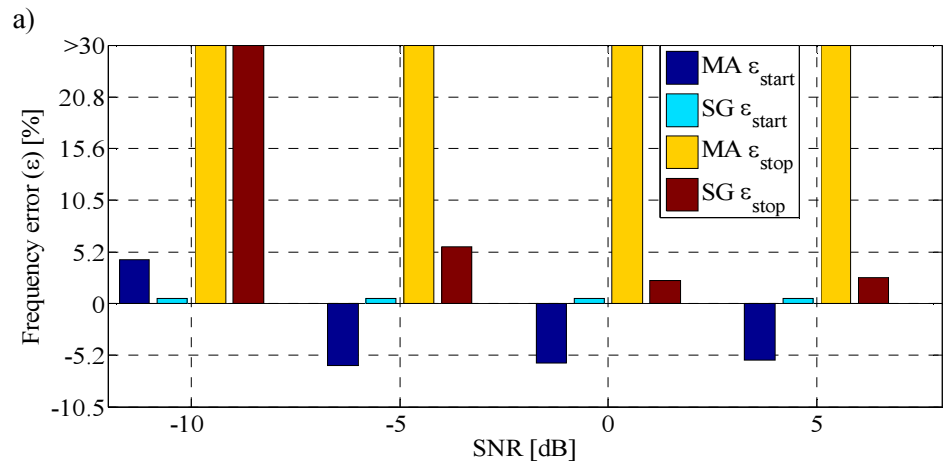

b)

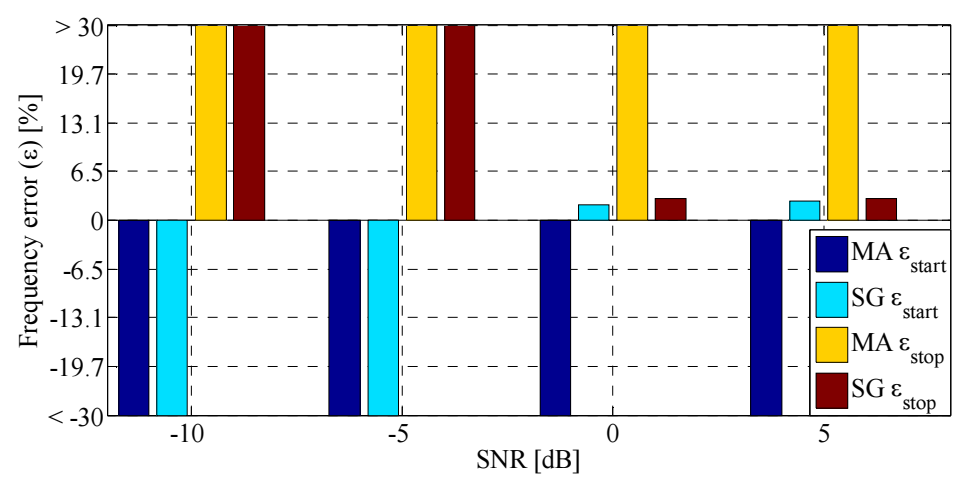

Fig. 14. The frequency error results for HYBRID scenario: a) a DVB-T transmitter; b) a wireless microphone. 
For the comments concerning Fig. 9a (for the simulation environment) are still valid for the corresponding Fig. 14a, with the difference that, in the emulation, the isolated case at $-5 \mathrm{~dB}$ where the MA signals were detected in their falling edge is absent here. So the tendency is confirmed with lower level performance due to the aforementioned emulation issues.

\section{Conclusions}

In this paper, a polynomial filtering (Savitzky-Golay) has been proposed to improve spectrum sensing performance in the VDSA approach for inter-vehicle communications in sub-GHz frequencies, using Cognitive Radio technology. The analyses in the simulation and emulation environment clearly confirm the goodness of this filtering method, especially if it is coupled with a spectrum sensing algorithm where edges are the main problem to detect and, consequently, their sharpness is fundamental for making it work properly. The trials have been performed for various SNR values.

For all considered SNR values (greater than $-10 \mathrm{~dB}$ ), the SG filtering is highly preferable to the MA approach, as explained in the previous sections, since it enables detection of users with smallers errors both in terms of bandwidth estimation and signal edges localization. In fact the SG provides errors of the same sign (almost always positive), and with a lower modulus. It is a critical benefit since the correct band detection enables to achieve efficiency in using opportunistic spectrum and also to prevent cognitive users to harmfully interfere with PU. Due to the computational burden of SG, its use is significantly restricted when medium/high SNR values are experienced.

The SNR value can be estimated with an a priori approach as follows:

1. Measure the noise power on a surely vacant channel (i.e. a reserved channel where no transmission is allowed).

2. Measure the (signal plus noise) power in the spectrum of interest.

3. Extract the signal power by considering a constant noise power (previously estimated).

4. Evaluate the SNR value.

The assumption that the noise power is constant in different channels is due to the hypothesis of AWGN, which represents an approximation of real conditions, but it remains widely used in the research community to reproduce noise variations in simulation trials.

Finally, it is worth to notice that, even if originally computationally heavy, the SG can be made lighter by a different implementation, as explained in the theoretical part of the presented paper, regarding the Savitzky-Golay filtering and the possibility of pre-implementing some operations prior to the filtering process.

\section{References}

[1] European Parliament and Council of the European Union (2010). Directive 2010/40/EU - on the framework for the deployment of Intelligent Transport Systems in the field of road transport and for interfaces with other modes of transport, Brussels, Belgium, 1-13.

[2] Chen, S. (2012). Vehicular Dynamic Spectrum Access: Using Cognitive Radio for Automobile Networks. Ph.D. Dissertation. Worcester Polytechnic Institute.

[3] Jiang, D., Delgrossi, L. (2008). IEEE 802.11p: Towards an international standard for wireless access in vehicular environments. Proc. of IEEE Vehicular Technology Conference VTC 2008, 2036-2040.

[4] Gozalvez, J., Sepulcre, M., Bauza, R. (2012). IEEE 802.11p vehicle to infrastructure communications in urban environments. IEEE Communications Magazine, 50(5), 176-183.

[5] Fernandez, J.A., Borries, K., Cheng, L., Kumar, B.V.K.V., Stancil, D.D., Bai, F. (2012). Performance of the 802.11p Physical Layer in Vehicle-to-Vehicle Environments. IEEE Transactions on Vehicular Technology, 61(1), 3-14. 
[6] Donato, E.A., Maia Menezes, J.G., Madeira, E.R.M., Villas, L.A. (2015). Impact of 802.11p Channel Hopping on VANET Communication Protocols. IEEE Latin America Transactions, 13(1), 315-320.

[7] Van de Beek, J., Riihijarvi, J., Achtzehn, A., Mähönen, P. (2012). TV White Space in Europe. IEEE Transactions on Mobile Computing, 11(2), 178-188.

[8] Taher, T. Bacchus, R., Zdunek, K., Roberson, D. (2011). Long-term spectral occupancy findings in Chicago. Proc. of 2011 IEEE Symposium on New Frontiers in Dynamic Spectrum Access Networks (DySPAN), 100107.

[9] López-Benítez, M., Casadevall, F. (2012). Improved energy detection spectrum sensing for cognitive radio. IET Communications, 6(8), 785-796.

[10] Narieda, S., Kageyama, T. (2013). Simple spectrum sensing techniques based on cyclostationarity detection in cognitive radio networks. Electronics Letters, 49(17), 1108-1109.

[11] Jiang, C., Li, Y., Bai, W., Yang, Y., Hu, J. (2012). Statistical matched filter based robust spectrum sensing in noise uncertainty environment. Proc. of 2012 IEEE 14th International Conference on Communication Technology, 1209-1213.

[12] Yucek, T., Arslan, H. (2009). A survey of spectrum sensing algorithms for cognitive radio applications. IEEE Communications Surveys \& Tutorials, 11(1), 116-130.

[13] Angrisani, L., Capriglione, D., Ferrigno, L., Miele, G. (2013). PSD estimation in cognitive radio systems: a performance analysis. Proc. of 19th IMEKO TC-4 Symposium Measurements of Electrical Quantities, 543548.

[14] Angrisani, L., Capriglione, D., Ferrigno, L., Miele, G. (2013). A measurement algorithm for frequency agility in cognitive radio. Proc. of 2013 IEEE International Instrumentation and Measurement Technology Conference, 371-376.

[15] Angrisani, L., Capriglione, D., Cerro, G., Ferrigno, L., Miele, G. (2014). The effect of Savitzky-Golay smoothing filter on the performance of a vehicular dynamic spectrum access method. Proc. of 20th IMEKO TC4, 1116-1121.

[16] Angrisani, L., Capriglione, D., Cerro, G., Ferrigno, L., Miele, G. (2014). Proposal and analysis of new algorithms for wideband spectrum sensing in cognitive radio. Proc. of 2014 IEEE International Instrumentation and Measurement Technology Conference, 701-706.

[17] Savitzky, A., Golay, M.J.E. (1964). Smoothing and differentiation of data by simplified least-squares procedures. Anal. Chem., 1627-1639.

[18] Schafer, R. (2011). What is a Savitzky-Golay filter? Lecture Notes. IEEE Signal Processing Magazine, 28(4), 111-117.

[19] Krishnan, S.R., Seelamantula, C.S. (2013). On the Selection of Optimum Savitzky-Golay Filters. IEEE Transactions on Signal Processing, 61(2), 380-391.

[20] Smith, S. (1997). The Scientist and Engineer's Guide to Digital Signal Processing. Moving Average filters. www.dspguide.com 